\title{
Evaluating the Performance of Hybrid Capture 2 Test as a Primary Screening Test from Studies Conducted in Low and Middle-Income Country Settings- Special Focus India
}

\author{
Kavita V Anand ${ }^{1}$, Sharmila A Pimple ${ }^{2 *}$, Atanu Bhattacharjee ${ }^{3}$, Gauravi A \\ Mishra $^{4}$, Surendra S Shastri ${ }^{5}$
}

\begin{abstract}
Among the screening tests for cervical cancer, advantages of screening with second generation molecular Hybrid Capture 2 (HC2) test is the high sensitivity and negative predictive value that makes it easy to implement as a cervical cancer screening policy necessitating less screening rounds. High income countries are now implementing HC2 test in their national cervical cancer screening program. Since the acceptance of any screening test depends on the sensitivity of the test, the current study was carried out to evaluate the sensitivity of HC2 test reported from Low- and Middle-income countries (LMIC) which share major burden of cervical cancer globally and to establish if HC2 test could be used as a primary screening test in India. Materials and methods: The population based cross sectional studies from LMICs which evaluated HC2 test as a primary screening modality to diagnose Cervical intraepithelial neoplasm grade 2 and above (CIN2+) lesions were included. Results: A total of 18 studies from LMIC involving 1,13,086 women were reviewed for sensitivity of HC2 as a primary screening test. The overall average sensitivity and specificity to diagnose CIN2+ lesions were 79.84\% (95\% CI-71.01,86.73) and 85.63\% (95\% CI- 84.37,86.92) respectively. India demonstrated an average sensitivity and specificity of $65 \%$ (95\% CI 57,77) and 93\% (95\% CI- 92,94) respectively. Conclusion: Results from LMIC demonstrate a comparably low sensitivity of HC2 test to diagnose CIN2+ lesions as compared to that reported from High income countries. Sensitivity of HC2 was substantially low for India. The current study discusses issues of HC2 assay and the role of untreated Reproductive tract infections as probable causes for low sensitivity of the test. This needs further research in an attempt to improve the sensitivity of the test in an era of self-sampling and low-cost HPV test on horizon to improve the coverage for cervical cancer.
\end{abstract}

Keywords: Human papilloma virus- HC2 test- HPV- cervical cancer screening- sexually transmitted infections

Asian Pac J Cancer Prev, 22 (8), 2709-2716

\section{Introduction}

The epidemiological studies have demonstrated the causal role of persistent oncogenic Human papillomavirus (HPV) infection as a main cause for cervical cancer (Walboomers et al., 1999; Bosch et al., 2002; IARC working group., 2007). Various screening tests aid in the diagnosis of precancerous stage of cervical cancer. The cytology-based screening demonstrated significant decrease in cervical cancer mortality rates for developed countries (High income countries) but similar results were not evident for Low- and middle-income countries (LMICs). The cytology-based screening has limitation in terms of sampling technique, transportation issues, interpretation skills of laboratory personnel (Lazcano-Ponce et al., 1999; WHO, 2006), and short screening intervals needed due to low sensitivity of the test reported (Aggarwal et al., 2010). Screening with Visual inspection with $5 \%$ acetic acid (VIA) is a proven feasible and cost-effective test for LMICs at present but it poses limitation in terms of the cost and time factors in training the health care providers, subjective biases in interpretation of test results and the burden on tertiary care centers due to its issues of false positive test results (Sankaranarayanan et al., 2003; Gaffikin et al., 2003; Shastri et al., 2014). The direct detection of HPV in cervical specimen by a molecular test offers an alternative for cytology/VIA based screening.

Among the molecular HPV tests available, the second-generation molecular Hybrid capture 2 tests (HC2, Qiagen, Gaithersburg), is a extensively validated test across different settings and identifies women at risk

${ }^{1}$ Homi Bhabha National Institute, Mumbai. India. ${ }^{2}$ Department of Preventive Oncology, Tata Memorial Centre, Mumbai. India. ${ }^{3}$ Department of Biostatistics, Centre for Cancer Epidemiology, Tata Memorial Centre. Mumbai. India. ${ }^{4}$ Department of Preventive Oncology, Tata Memorial Centre, Mumbai, India. ${ }^{5}$ Department of Health Disparities Research, Division of Cancer Prevention \& Population Science, M.D Anderson Cancer Centre, Houston,USA. *ForCorrespondence: drsharmilapatil@yahoo.com 
of developing cervical cancer, needing a close follow up. The test has probes for all 13 recognised oncogenic HPV types, reported worldwide (Malloy et al., 2000; Qiagen, 2008; Bruni et al., 2010). It becomes easier to implement HPV based screening program due to its advantages (WHO, 2006; Quigen, 2008) - high sensitivity reported from High income countries, safe longer screening interval if tested negative, minimal manpower for performing the test, the potential of self-collection, the reproducibility and standardization of the test with no inter and intra subjective variability.

HPV testing is now a recommended screening test for cervical cancer (IARC, 2005). From emerging evidences, the High-income countries have now shifted from cytology to molecular $\mathrm{HC} 2$ based screening in their national screening programs for cervical cancer (Malloy et al., 2000; Cuzick et al., 2008; Arbyn et al., 2010) while LMICs are still contemplating on using HPV test for cervical cancer screening for their country. There are encouraging results from well conducted population-based trials from LMICs like India (Sankaranarayanan et al., 2009) and Mexico (Flores et al., 2003) demonstrating that, a simple and reliable molecular $\mathrm{HC} 2$ test which is now available even in low-income countries has a potential to be accepted as primary screening tool in the near future.

Since the potential of any screening test to be adopted by a country depends on the sensitivity and specificity (test characteristics), the aim of current review was to appraise the test characteristics of $\mathrm{HC} 2$ as a primary cervical cancer screening test reported from LMICs, especially from Indian context.

\section{Materials and Methods}

\section{Inclusion criteria}

1] Population-based, cross-sectional studies from LMICs which evaluated the test characteristics of second generation of $\mathrm{HC} 2$ test as a primary screening test to diagnose high grade precancerous lesion of cervix on histopathology (CIN2+ lesion). 2] The cervical samples collected by Physician/ trained health care workers. 3] The studies using the value of 1 and above Relative light unit (RLU) corresponding to 5000 or more viral copies per $\mathrm{ml}$ as a cut off ratios for interpretation of a positive test results. 4] The gold standard verification was either colposcopy or colposcopy directed biopsy to diagnose severity of the diseases.

\section{Exclusion criteria}

1] Studies providing data on test characteristics of $\mathrm{HC} 2$ as a triage test, on self-collected cervical samples or among high-risk population (HIV or Sex workers or any other immunocompromised population). 2] Studies using other molecular HPV test including signal amplification test (Care HPV) as a primary screening test as these tests are currently limited to research settings. 3] The studies using first-generation Hybrid capture test ( $\mathrm{HC1}$ ) since the test lacks all the probes of recognised oncogenic HPV till date. 4] Studies not reporting respective Confidence Intervals (CI) for the reported test characteristics of HC2 assay. 5] Studies reporting the test characteristics of $\mathrm{HC} 2$ test using cytology as a gold standard. 6] Hospital based studies, as women are expected to be high risk for precancerous or cancerous lesions and may not reflect the true scenario.

\section{Data sources and searches}

This paper is based on information gathered from published peer-reviewed articles on cervical cancer screening in PubMed data base from year 2000 to 2019 since majority of articles on molecular HPV testing were published after year 2000. The keywords and their corresponding MeSH term used to build up search strategy were 'cervical cancer screening' OR 'cervical intraepithelial neoplasm' OR 'cervical dysplasia' AND 'human Papillomavirus' OR 'HPV test' OR 'HC2 test' NOT 'HPV vaccination' NOT 'HPV self-sampling' We examined bibliographies of relevant articles to identify additional references. Journal articles fulfilling the inclusion criteria were included in the study.

Figure 1 - The search strategy identified 459 articles which were screened for appropriateness to the study topic. The bibliography of relevant article was screened for additional references. A total of 56 studies were identified that used molecular HC2 test (Qiagen) as a primary screening test for cervical cancer. These studies reported the test characteristics of $\mathrm{HC} 2$ as a standalone test AND as a comparator test to other recognized screening modalities for cervical cancer and other molecular HPV tests. These articles were then reviewed for the study settings. Total of 38 studies were excluded with reasons - 22 studies were clearly excluded as articles did not fulfill the inclusion criteria for study settings. 16 studies from LMICs were excluded with reasons: Studies reported test characteristic of HC2 on hospital based data (Sodhani et al., 2006; Ma et al., 2010; Bhatla et al., 2012), study had no clarity on type of settings (Katyal et al.,2011), studies reported estimates of HC2 on CIN3 + lesion only (Moy et al., 2010; Belinson et al., 2011; Nieves et al., 2013), studies mentioned no estimates of test characteristics of $\mathrm{HC} 2$ test or CI intervals (Schiffman et al., 2000; Sankaranarayanan et al., 2009; Cagle et al., 2010; Li et al., 2010; Girianelli et al., 2006), duplicate data, cytology as gold standard (Belinson et al., 2001; Blumenthal et al., 2001; Longatto-Filho et al., 2012; Katanga et al., 2019). Total 18 population based cross-sectional studies fulfilling the eligibility criteria were included in the analysis.

\section{Statistical analysis}

The published test characteristic of $\mathrm{HC} 2$ as a primary screening test from the LMIC were used to calculate weightage average sensitivity and specificity of the $\mathrm{HC} 2$ test. Separate analysis was done to demonstrate the test characteristics of $\mathrm{HC} 2$ from Indian context as a primary focus of the study. The weightage average of sensitivity, specificity, $95 \%$ CI of sensitivity and specificity were analyzed and forest plots were constructed using $\mathrm{R}$ software.

\section{Results}

Table 1: The Table summarizes characteristics of population based, cross-sectional studies from LMICs, 
Table 1. Summary of Studies from Low- and Middle-Income Countries that Reported the Test Characteristic of HC2 Test as a Primary Screening Test to Diagnose CIN2+ Lesions

\begin{tabular}{|c|c|c|c|c|c|c|}
\hline & Country & $\begin{array}{l}\text { Age of } \\
\text { women }\end{array}$ & $\begin{array}{l}\text { Population } \\
\text { size }\end{array}$ & Sensitivity $(95 \% \mathrm{CI})$ & Specificity $(95 \% \mathrm{CI})$ & Ref \\
\hline \multicolumn{7}{|c|}{ Low-income countries } \\
\hline 1 & Zimbabwe & $25-55$ & 2140 & $81 \%(75-86)$ & $62 \%(59-64)$ & Womack.,2000 \\
\hline 2 & South Africa & $35-65$ & 424 & $88.4 \%(76.9-81.9)$ & $81.9 \%(76.5-86.5)$ & Kuhn.,2000 \\
\hline \multirow[t]{2}{*}{3} & Democratic republic of congo & $30 \&$ above & 1528 & $83.4 \%(66.8-100)$ & $90.8 \%(89.0-92.7)$ & Mahmud.,2011 \\
\hline & Average $^{\mathrm{F}}$ & & & $82.69 \%(72.13-90.8)$ & $74.81 \%(72.01-77.04)$ & \\
\hline \multicolumn{7}{|c|}{ Low middle-income countries } \\
\hline \multirow[t]{4}{*}{4} & India* & $25-65$ & 3555 & $50 \%(36.6-63.4)$ & $91.7 \%(90.7-92.6)$ & Sankaranarayanan et al.,2004 \\
\hline & India* & $25-65$ & 6568 & $71.7 \%(58.6-82.6)$ & $94.5 \%(93.9-95.0)$ & Sankaranarayanan et al.,2004 \\
\hline & India** & $25-65$ & 3474 & $70.5 \%(57.4-81.5)$ & $93.6 \%(92.7-94.4)$ & Sankaranarayanan et al.,2004 \\
\hline & India $* * *$ & $25-65$ & 4488 & $80 \%(67.7-89.2)$ & $94.6 \%(93.9-95.3)$ & Sankaranarayanan et al.,2004 \\
\hline 5 & India & $30-65$ & 3407 & $62 \%(47.2-75.4)$ & $93.5 \%(92.6-94.3)$ & Shastri et al.,2005 \\
\hline 6 & Vanuatu & $30-50$ & 514 & $81 \%(61-93)$ & $94 \%(91-95)$ & McAdam et al.,2010 \\
\hline 7 & India & $25-60$ & 2331 & $61.2 \%(38.5-79.95)$ & $91.0 \%(90.5-91.5)$ & Gravitt et al.,2010 \\
\hline \multirow[t]{2}{*}{8} & India & $30-60$ & 39,740 & $64.4 \%(57.6-71.0)$ & $97 \%(96.8-97.1)$ & Basu et al.,2015 \\
\hline & Average $^{\mathrm{T}}$ & & & $65.66 \%(56.01-74.34)$ & $95.66 \%(95.23-95.99)$ & \\
\hline \multicolumn{7}{|c|}{ Upper middle-income countries } \\
\hline 9 & Mexico & $15-85$ & 7868 & $90.7 \%(83.4-95.0)$ & $93.2 \%(92.1-93.3)$ & Salmeron etal.,2003 \\
\hline 10 & China & $35-45$ & 1836 & $95.2 \%(88.1-98.7)$ & $85.9 \%(84.1-87.5)$ & Pan et al.,2003 \\
\hline 11 & China & $35-50$ & 8497 & $96.8 \%(95.0-98.6)$ & $79.7 \%(78.9-80.5)$ & Belinson et al.,2003 \\
\hline 12 & Latin America & $18-60$ & 4195 & $82.8 \%(76.3-88.4)$ & $86.4 \%(85.3-87.5)$ & Sarian et al.,2005 \\
\hline 13 & Peru & $25-49$ & 5435 & $77.3 \%(70.4-83.5)$ & $89.3 \%(88.5-90.1)$ & Almonte.,2007 \\
\hline 14 & China & $30-54$ & 2388 & $97.1 \%(93.2-100.0)$ & $85.6 \%(84.2-87.1)$ & Qiao et al.,2008 \\
\hline 15 & China & $15-59$ & 2562 & $90.4 \%(83.3-94.7)$ & $86.4 \%(85.0-87.7)$ & Li et al.,2009 \\
\hline 16 & China & $25-59$ & 2090 & $88.9 \%(70.8-97.6)$ & $84.5 \%(82.8-86.1)$ & Wu et al.,2010 \\
\hline 17 & China & $16-54$ & 2625 & $97.9 \%(88.7-100)$ & $90.2 \%(90.0-91.3)$ & Belison et al.,2012 \\
\hline \multirow[t]{3}{*}{18} & China & $25-65$ & 7421 & $95.8 \%(91.2-98.5)$ & $87.1 \%(86.3-87.9)$ & Zhao et al.,2013 \\
\hline & Average $^{\mathrm{T}}$ & & & $91.18 \%(85.07-95.06)$ & $86.41 \%(85.86-87.71)$ & \\
\hline & Grand average $^{\mathrm{T}}$ & & & $79.84 \%(71.07-86.73)$ & $85.63 \%(84.37-86.92)$ & \\
\hline
\end{tabular}

${ }^{\mp}$, Weighted average; Among the studies included, 1 Indian multicentric study reported the test characteristics of HC2 test across 4 different centres in 3 locations, Chittaranjan National Cancer Centre, Kolkata 1,2*, Tata Memorial Hospital; Mumbai**, Regional Cancer Centre; Trivandrum***

which evaluated second generation $\mathrm{HC} 2$ test as a primary cervical cancer screening modality to diagnose CIN2+ lesion. A total of 18 studies from LMIC involving 113086 women were reviewed for test characteristics of $\mathrm{HC} 2$ as a primary screening test. The overall weightage average sensitivity of $79.84 \%(95 \%$ CI-71.01,86.73) and specificity of $85.63 \%$ (95\% CI- $84.37,86.92)$ to diagnose CIN2+ lesions was demonstrated for LMIC. There are limited population based cross- sectional studies from low-income countries which evaluated the performance of $\mathrm{HC} 2$ test as a primary screening test. The calculated average sensitivity and specificity reported for the countries was $82.69 \%(95 \%$ CI-72.13,90.8) and $74.81 \%$ (95\% CI- 72.01,77.04). Among the Middle-income countries, majority of studies are from India and China. Within the Middle-income countries, there is a significant difference in sensitivity of $\mathrm{HC} 2$ test demonstrated between Upper and Lower middle-income countries. The upper middle-income countries demonstrated a high accuracy of HC2 to diagnose CIN2+ lesion, $91.18 \%$ (95\% CI- 85.07,95.06) as compared to $65.66 \%(95 \%$ CI- 56.01,74.34) demonstrated for lower middle-income countries, while specificity reported were $86.41 \%$
(95\% CI- 85.86,87.71) and 95.66\% (CI-95.23,95.99) respectively.

Figure 2: A total of 7 cross-sectional, populationbased studies from India involving 63,563 women were reviewed for test characteristics of $\mathrm{HC} 2$ test. The age of the women ranged from 25- 60 years, where increase chances of persistence HPV infection is demonstrated. The average weighted sensitivity and specificity demonstrated was 65\% (95\% CI- 57,77) and 93\% (95\% CI- 92,94) respectively.

\section{Discussion}

The current study focused on HC2 (Qiagen) as a primary screening test as the test is widely evaluated and a recommended test for cervical cancer screening. Since the primary focus of any screening test is to diagnose the lesion at early stage, we analysed data using only CIN2+ lesions as endpoint as it provides more time frame to treat patients effectively if diagnosed than CIN3+ lesions particularly in resource constrain countries. There is a difference in sensitivity demonstrated in the studies from LMICs and those reported from High income countries. 


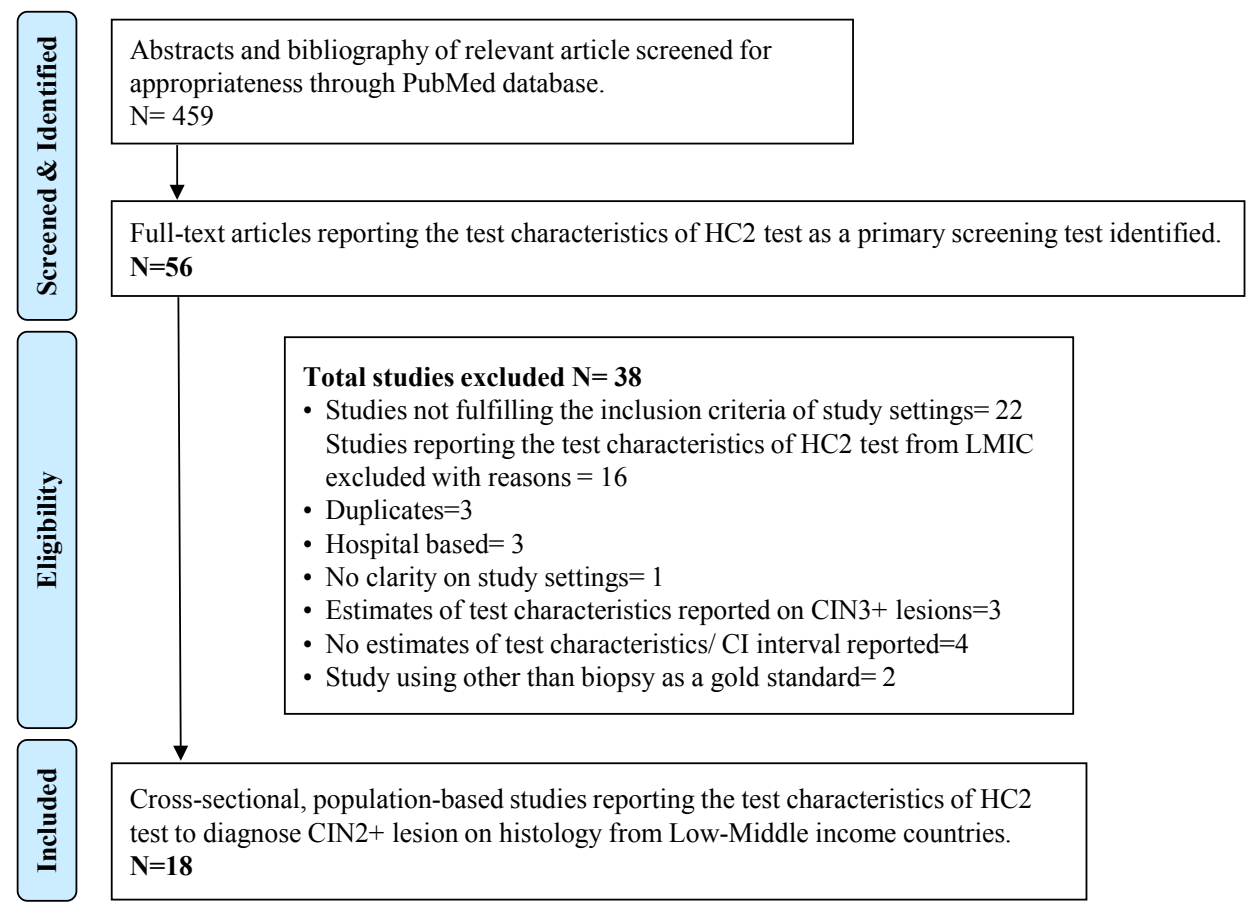

Figure 1. Search Flow Chart. LMICs, Low- and Middle-income countries.

The studies from Europe and America which evaluated the $\mathrm{HC} 2$ test as a primary screening test reported sensitivity and specificity estimates of $97.9 \%$ (95\% CI- 95.9\%- 99.9\%) and 91.3\% (95\% CI- $89.5 \%$ $-93.1 \%)$ respectively for $\mathrm{HC} 2$ test to diagnose $\mathrm{CIN} 2+$ lesions (Arbyn et al., 2006). The similar findings of high sensitivity (96\%) of $\mathrm{HC} 2$ assay were also reported from

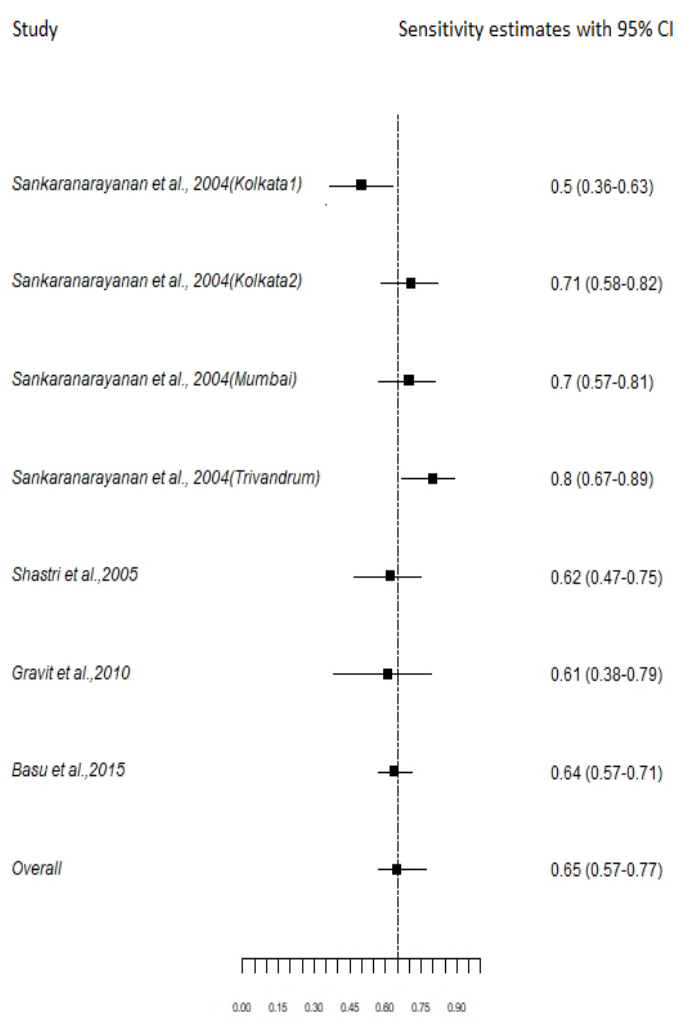

these countries by Arbyn $\mathrm{M}$ et al in their meta-analysis (Arbyn et al., 2012). These results support HC2 test as a primary screening test for cervical cancer.

The above findings are inconsistent with the results demonstrated from LMICs. The overall average sensitivity and specificity demonstrated for the test was $79.84 \%$ and $85.63 \%$ respectively (Table 1). Among the LMICs, the test

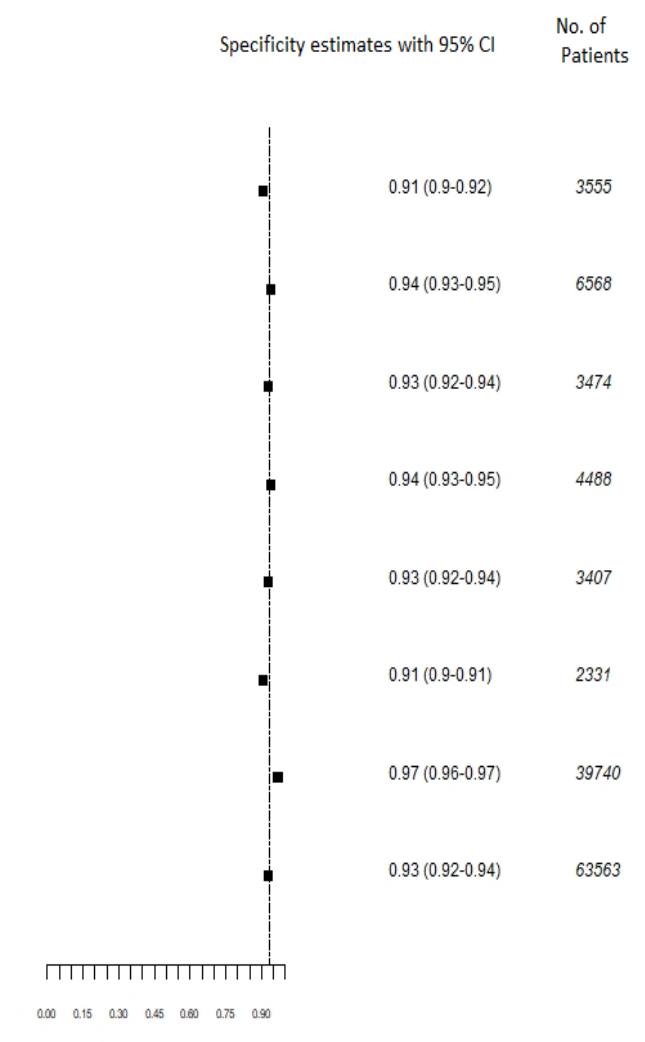

Figure 2. Population Based Cross-Sectional Studies from India Reporting the Test Characteristics of HC2 Test as a Primary Screening Test to Diagnose CIN2+ Lesions 
was widely evaluated in China and India. Studies from China report the test characteristics comparable to High income countries. The probable reasons for low sensitivity of HC2 test reported from other LMICs countries are - improper sampling techniques (Sankaranarayanan et al., 2004) degradation of HPVDNA due to temperature fluctuations during transportation and storage (Almonte, 2007) prevalence of HPV in a geographical area, RLU/ $\mathrm{CO}$ cut off recommended for interpretation of positive test results for respective countries (Womack et al., 2000) and prevalence of HPV type in a particular geographical area not included in probe cocktail of HC2 assay (Arbyn et al., 2008). Among the data of sensitivity of HC2 test presented in the current review, though South Africa (Kuhn et al., 2000) reported a good sensitivity of $88.4 \%$, the main limitations of this study were verification biases, small retrospective sub- grouped sample tested with HC2 test.

The studies from India were evaluated separately for test characteristics of $\mathrm{HC} 2$ test as a primary screening test (Figure 2). These studies demonstrated homogeneity for age as they enrolled women in age group of 25-65 years where persistence of HPV infection is demonstrated. All studies accounted for verification bias, as all women enrolled in the study underwent colposcopy and biopsy were taken for cervical abnormalities except one study from Kolkata which reported the test characteristics after adjusting for verification biases (Basu et al., 2015) The average sensitivity and specificity of HC2 test demonstrated for India was 65\% (95\% CI- 57, 77) and $93 \%(95 \%$ CI-92, 94) respectively which is substantially very low as compared to other countries.

A study conducted by Bruni et al., (2010) on a million women with normal cytological findings across the world to detect prevalence of cervical HPV by molecular HPVDNA test, the author mentioned, though the prevalence rate of cervical HPV were lower for India, the country reports high incidence rates of cervical cancer. This comment suggests a possibility of missing HPV infections by molecular HPV test leading to false negative test results. According to the published literature (Burd et al., 2003) the false negative test results of HC2 test is estimated to be $7.5 \%$. Interference of contraceptive jelly, vaginal pessaries, vaginal douches are reported to hamper the diagnostic performance of the tests. Though HC2 test is the most validated test across various settings, main limitation of the test is lack of inbuilt mechanism to monitor cell adequacy. The assay needs 5000 viral copies in a cervical sample to be interpreted as a positive test. The test result 'HPV not detected' opens up a debate, whether the cervical sample is truly negative for HPV or the viral load is less than the detection ratio of the test assay due to less cells collected (Malloy at al., 2000; Jastania et al., 2006). There has always been a concern about blood and mucous associated with reproductive tract infections (RTIs) interfering with the sensitivity of screening test for cervical cancer (Sasieni et al., 2003; Sankaranarayanan et al., 2004). The concomitant STIs affecting the test performance of HPV testing has also been commented (Cuzick et al., 2006). A prospective study (Liu et al., 2013) which estimated the prevalence of HPV genotype among three groups of women; group 1-women with mucopurulent cervicitis, group 2- healthy women with no cervicitis and group 3- women with Invasive cancer, the author reported $10 \%$ higher failure rates to extract cervical HPV DNA among women with cervicitis. This finding demonstrates the potential of HPVDNA test to be influenced by cervicitis caused by RTIs.

India reports a huge burden of undiagnosed and untreated RTIs infections (Durai et al., 2019). The prevalence is expected to be high due to illiteracy, ignorance, social stigma, cultural norms, lack of quality health care facilities for screening and treating RTIs and poor access to health care facilities, if available (Garg et al., 2001; Prasad et al., 2005; Ray et al., 2008). At present there are gaps in literature regarding the burden of RTIs induced cervicitis among Indian population. To our knowledge, the only study reported the prevalence of cervicitis to be $55 \%$ in Indian population (Tribhovandas et al., 2013). The RTIs caused by bacterial microbes and the genital HPV infections are common among women in reproductive age group and both infections are sexually transmitted. Literature supports, cervicitis (inflammation) associated with RTIs to serves as a co-factor for acquisition and persistence of HPV infection among women. Inflammation modulates the progression of HPV infection to precancerous and cancerous lesion of cervix (Castle et al., 2003; Woodman et al., 2007; Williams et al., 2011). These group of women with concomitant infections (RTIs and HPV) needs prompt detection of HPV along effective treatment of RTIs.

With the prospect of using low-cost HPV test as a primary screening test in near future the data of $\mathrm{HC} 2$ as a primary screening test from India is warned. In the present review the population based cross- sectional studies from India reported a substantial low estimated of sensitivity of $\mathrm{HC} 2$ test. We assume that, RTIs among women resulting in mucopurulent discharge are likely to hamper the detection rates of cervical HPV in women with concomitant infection due to cell inadequacy. This may lead to less viral copies below the detection threshold of HC2 test assay resulting in false negative test results which has a potential to affect the sensitivity of the test. At a country level, literature supports HPV based screening program, primarily due to benefits of preventing the repetitive screening costs which can burden the already strained health budget of LMIC. With ongoing research on molecular HPV assay on self-sampling modalities in anticipation to increase the coverage of cervical cancer screening and introduction of care HPV test (Qiagen) that can be conducted on field which are supposed to be low cost, adds to the advantages of screening strategy based on molecular HPV testing as a stand-alone test. Considering the results of the present study and above discussion centered to specific issues with $\mathrm{HC} 2$ assay, it becomes relevant to evaluate the effect of mucopurulent discharge due to RTIs on HC2 assay. If the suggested hypothesis is proven, then it would have an important public health implication of treating cervicitis before performing $\mathrm{HC} 2$ test in an attempt to reduce the false negative test results. 


\section{Author Contribution Statement}

None.

\section{Acknowledgments}

The current review has been carried out as a part of main study (Clinical Trial.gov no- NCT02830230) financially supported by Intramural funding of Research Administration Council, Tata Memorial Centre, Grant No 1671 .

\section{Conflict of Interest disclosure}

This manuscript has been read and approved by all the authors and represents honest work. The Authors declare there is no conflict of interest.

\section{References}

Aggarwal P, Batra S, Gandhi G, Zutshi V (2010). Comparison of Papanicolaou test with visual detection tests in screening for cervical cancer and developing the optimal strategy for low resource settings. Int J Gynecol Cancer, 20, 862-8.

Arbyn M, Anttila A, Jordan J, et al (2010). European guidelines for quality assurance in cervical cancer screening. - summary document. Ann Oncol, 21, 448-8.

Arbyn M, Ronco G, Anttila A, et al (2012). Evidence regarding human papillomavirus testing in secondary prevention of cervical cancer. Vaccine, 30, F88-9.

Arbyn M, Sankaranarayanan R, Muwonge R, et al (2008). Pooled analysis of the accuracy of five cervical cancer screening tests assessed in eleven studies in Africa and India. Int $J$ Cancer, 123, 153-60.

Arbyn M, Sasieni P, Meijer CJ, et al (2006). Clinical applications of HPV testing: a summary of meta-analyses. Vaccine, 24, S78-9.

Basu P, Mittal S, Banerjee D, et al (2015). Diagnostic accuracy of VIA and HPV detection as primary and sequential screening tests in a cervical cancer screening demonstration project in India. Int $J$ Cancer, 137, 859-7.

Belinson JL, Du H, Yang B, et al (2012). Improved sensitivity of vaginal self-collection and high-risk human papillomavirus testing. Int J Cancer, 130, 1855-60.

Belinson JL, Qiao YL, Pretorius RG, et al (2003). Shanxi Province cervical cancer screening study II: self-sampling for high-risk human papillomavirus compared to direct sampling for human papillomavirus and liquid based cervical cytology. Int J Gynecol Cancer, 13, 819-6.

Belinson JL, Wu R, Belinson SE, et al (2011). A population-based clinical trial comparing endocervical high-risk HPV testing using hybrid capture 2 and Cervista from the SHENCCAST II Study. Am J Clin Pathol, 135, 790-5.

Bhatla N, Puri K, Kriplani A, et al (2012). Adjunctive testing for cervical cancer screening in low resource settings. Australian New Zealand J Obstet Gynaecol, 52, 133-9.

Blumenthal P, Gaffikin L, Chirenje ZM, et al (2001). Adjunctive testing for cervical cancer in low resource settings with visual inspection, HPV, and the Pap smear. Int J Gynaecol Obstet, 72, 47-3.

Bosch FX, Lorinex A, Munoz N, et al (2002). The causal relation between human papillomavirus and cervical cancer. $J$ Clin Pathol, 55, 244-5.

Bruni L, Diaz M, Castellsagué M, et al (2010). Cervical human papillomavirus prevalence in 5 continents: meta-analysis of 1 million women with normal cytological findings. $J$ Infect
Dis, 202, 1789-9.

Burd EM (2003). Human papillomavirus and cervical cancer. Clin Microbiol Rev, 16, 1-7.

Cagle AJ, Hu SY, Sellors JW, et al (2010). Use of an expanded gold standard to estimate the accuracy of colposcopy and visual inspection with acetic acid. Int J Cancer, 126, 156-1.

Castle PE, Giuliano AR (2003). Chapter 4: Genital tract infections, cervical inflammation, and antioxidant nutrientsassessing their roles as human papillomavirus cofactors. JNCI Monographs, 2003, 29-4.

Cuzick J, Clavel C, Petry KU, et al (2006). Overview of the European and North American studies on HPV testing in primary cervical cancer screening. Int J Cancer, 119, 1095-1.

Cuzick J, Arbyn M, Sankaranarayanan R, et al (2008). Overview of human papillomavirus-based and other novel options for cervical cancer screening in developed and developing countries. Vaccine, 26, K 29-1.

Durai V, Varadharajan S, Muthuthandavan AR (2019). Reproductive tract infections in rural India-A populationbased study. J Family Med Prim Care, 8, 3578-3.

Flores Y, Bishai D, Lazcano E, et al (2003). Improving cervical cancer screening in Mexico: results from the Morelos HPV Study. Salud Publica Mex, 45, S388-9.

Gaffikin L, Lauterbach M, Blumenthal PD (2003). Performance of visual inspection with acetic acid for cervical cancer screening: a qualitative summary of evidence to date. Obstet Gynecol Surv, 58, 543-5.

Garg S, Bhalla P, Sharma N, et al (2001). Comparison of selfreported symptoms of gynecological morbidity with clinical and laboratory diagnosis in a New Delhi slum. Asia Pac Popul J, 16, 75-2.

Gravitt PE, Paul P, Katki HA, et al (2010). Effectiveness of VIA, Pap, and HPV DNA testing in a cervical cancer screening program in a peri-urban community in Andhra Pradesh, India. PLoS One, 5, e13711.

IARC (2005). Handbook of cancer prevention. Vol 10: Cervix cancer screening, Lyon, International Agency for Research on cancer.

IARC Working Group on the Evaluation of Carcinogenic Risks to Humans (2007). Human papillomaviruses. IARC Monographs on the evaluation of carcinogenic risk to Humans, 90, pp 1-636.

Jastania R, Geddie WR, Chapman W, Boerner S (2006). Characteristics of apparently false-negative digene hybrid capture 2 high-risk HPV DNA testing. Am J Clin Pathol, 125, 223-8.

Katyal S, Mehrotra R (2011). Complementary procedures in cervical cancer screening in low resource settings. $J$ Obstet Gynaecol India, 61, 436-8.

Lazcano-Ponce EC, Moss S, de Ruíz PA, Castro JS, Avila MH (1999). Cervical cancer screening in developing countries: why is it ineffective? The case of Mexico. Arch Med Res, 30, 240.

Li C, Wu M, Wang J, et al (2010). A population-based study on the risks of cervical lesion and human papillomavirus infection among women in Beijing, People's Republic of China. Cancer Epidemiol Biomarkers Prev, 19, 2655-4.

Li N, Shi JF, Franceschi S, Plummer M (2009). Different cervical cancer screening approaches in a Chinese multicentre study. Br J Cancer, 100, 532-7.

Liu W, Wu EQ, Yu XH, et al (2013). Detection of human papillomavirus genotypes associated with mucopurulent cervicitis and cervical cancer in Changchun, China. Int $J$ Gynaecol Obstet, 120, 124-6.

Longatto-Filho A, Naud P, Derchain SF, et al (2012). Performance characteristics of Pap test, VIA, VILI, HRHPV testing, cervicography, and colposcopy in diagnosis of 
significant cervical pathology. Virchows Archiv, 460, 577-5.

Belinson J, Qiao YL, Pretorius R, et al (2001). Shanxi Province Cervical Cancer Screening Study: a cross-sectional comparative trial of multiple techniques to detect cervical neoplasia. Gynecol Oncol, 83, 439-4.

Katanga J, Kjaer SK, Manongi R, et al (2019). Performance of care HPV, hybrid capture 2 and visual inspection with acetic acid for detection of high-grade cervical lesion in Tanzania: A cross-sectional study. PLoS One, 14, e0218559.

Girianelli VR, Thuler LC, Szklo M, et al (2006). Comparison of human papillomavirus DNA tests, liquid-based cytology and conventional cytology for the early detection of cervix uteri cancer. Eur J Cancer Prev, 15, 504-0.

Womack SD, Chirenje ZM, Blumenthal PD, et al (2000). Evaluation of a human papillomavirus assay in cervical screening in Zimbabwe. BJOG, 107, 33-8.

Kuhn L, Denny L, Pollack A, et al (2000). Human papillomavirus DNA testing for cervical cancer screening in low-resource settings. $J$ Natl Cancer Inst, 92, 818-5.

Mahmud SM, Sangwa-Lugoma G, Nasr SH, et al (2012). Comparison of human papillomavirus testing and cytology for cervical cancer screening in a primary health care setting in the Democratic Republic of the Congo. Gynecol Oncol, 124, 286-1.

Ma L, Bian ML, Cheng JY, et al (2010). Hybrid capture II for high-risk human papillomavirus DNA testing to detect cervical precancerous lesions: a qualitative and quantitative study. Exp Ther Med, 1, 193-8.

Malloy C, Sherris J, Herdman C (2000). HPV/DNA testing: technical and programmatic issues for cervical cancer prevention in low-resource settings. Path, 2000, 1-24.

Moy L, Zhao F, Li L, et al (2010). Human papillomavirus testing and cervical cytology in primary screening for cervical cancer among women in rural China: comparison of sensitivity, specificity, and frequency of referral. Int $J$ Cancer, 127, 646-6.

Nieves L, Enerson CL, Belinson S, et al (2013). Primary cervical cancer screening and triage using an mRNA human papillomavirus assay and visual inspection. Int J Gynecol Cancer, 23, 513- 8.

Othman NH, Rebolj M (2009). Challenges to cervical cancer screening in a developing country: The case of Malaysia. Asian Pac J Cancer Prev, 10, 747-1.

Pan Q, Belinson JL, Li L, et al (2003). A thin-layer, liquidbased pap test for mass screening in an area of China with a high incidence of cervical carcinoma. A cross-sectional, comparative study. Acta Cytologica, 47, 45-0

Prasad JH, Abraham S, Kurz KM, et al (2005). Reproductive tract infections among young married women in Tamil Nadu, India. Int Fam Planning Perspect, 31, 73-2.

Qiagen (2008). hc2 HIGH-RISK TEST. Qiagen Gaithersburg, Inc.US.

Qiao YL, Sellors JW, Eder PS, et al (2008). A new HPV-DNA test for cervical-cancer screening in developing regions: a cross-sectional study of clinical accuracy in rural China. Lancet Oncol, 9, 929-6.

Ray K, Bala M, Bhattacharya M, et al (2008). Prevalence of RTI/STI agents and HIV infection in symptomatic and asymptomatic women attending peripheral health set-ups in Delhi, India. Epidemiol Infect, 136, 1432.

Salmeron J, Lazcano-Ponce E, Lorincz A, et al (2003). Comparison of HPV-based assays with Papanicolaou smears for cervical cancer screening in.Morelos State, Mexico. Cancer Causes Control, 14, 505-2.

Sarian LO, Derchain SF, Naud P, et al (2005). Evaluation of visual inspection with acetic acid (VIA), Lugol's iodine (VILI), cervical cytology and HPV testing as cervical
HC2 Test as a PrimaryScreening Test in Indian Settings

screening tools in Latin America: This report refers to partial results from the LAMS (Latin American Screening) study. J Med Screen, 12, 142-9.

Almonte M, Ferreccio C, Winkler JL, et al (2007). Cervical screening by visual inspection, HPV testing, liquid-based and conventional cytology in Amazonian Peru. Int J Cancer, 121, 796-2.

Sankaranarayanan R, Chatterji R, Shastri SS, et al (2004). Accuracy of human papillomavirus testing in primary screening of cervical neoplasia: results from a multicenter study in India. Int J Cancer, 112, 341-7.

Sankaranarayanan R, Nene BM, Shastri SS, et al (2009). HPV screening for cervical cancer in rural India. $N$ Engl J Med, 360, 1385-4.

Sankaranarayanan R, Thara S, Sharma A, et al (2004). Multicentre Study Group on Cervical Cancer Early Detection in India. Accuracy of conventional cytology: results from a multicentre screening study in India. J Med Screen, 11, 77-4.

Sankaranarayanan R, Wesley R, Thara S, et al, (2003). Test characteristics of visual inspection with $4 \%$ acetic acid (VIA) and Lugol's iodine (VILI) in cervical cancer screening in Kerala, India. Int $J$ Cancer, 106, 404-8.

Sasieni P, Adams J, Cuzick J (2003). Benefit of cervical screening at different ages: evidence from the UK audit of screening histories. Br J Cancer, 89, 88- 3.

Schiffman M, Herrero R, Hildesheim A, et al (2000). HPV DNA testing in cervical cancer screening: results from women in a high-risk province of Costa Rica. JAMA, 283, 87-3.

Shastri SS, Dinshaw K, Amin G, et al (2005). Concurrent evaluation of visual, cytological and HPV testing as screening methods for the early detection of cervical neoplasia in Mumbai, India. Bull World Health Organ, 83, 186-4.

Shastri SS, Mittra I, Mishra GA, et al (2014). Effect of VIA screening by primary health workers: randomized controlled study in Mumbai, India. J Natl Cancer Inst, 106, dju009. https://doi.org/10.1093/jnci/dju009.

Shi JF, Canfell K, Lew JB, Qiao YL (2012). The burden of cervical cancer in China: synthesis of the evidence. Int $J$ Cancer, 130, 641-2.

Sodhani P, Gupta S, Sharma JK, et al (2006). Test characteristics of various screening modalities for cervical cancer: a feasibility study to develop an alternative strategy for resource-limited settings. Cytopathology, 17, 348-2.

Tribhovandas PM, Mansukhbhai SH, Vinodkumar GV (2013). A study of prevalence of sexually transmitted infections \& response to syndromic treatment among married women of reproductive age group in rural area of Parol Primary Health Centre under Thane district, Maharashtra, India. GJMEDPH, 2, 1-7.

Walboomers JM, Jacobs MV, Manos MM, et al (1999). Human papillomavirus is a necessary cause of invasive cervical cancer worldwide. J Pathol, 189, 12-9.

Williams VM, Filippova M, Soto U, Duerksen-Hughes PJ (2011). HPV-DNA integration and carcinogenesis: putative roles for inflammation and oxidative stress. Future Virol, 6, 45-7.

Woodman CB, Collins SI, Young LS (2007). The natural history of cervical HPV infection: unresolved issues. Nat Rev Cancer, 7, 11-2.

World Health Organization (2006). Comprehensive Cervical Cancer Control. A guide to essential practice. Geneva; WHO.

Wu R, Belinson SE, Du H, et al (2010). Human papillomavirus messenger RNA assay for cervical cancer screening: the Shenzhen Cervical Cancer Screening Trial I. Int J Gynecol Cancer, 20, 1411- 4.

Zhao FH, Jeronimo J, Qiao YL, et al (2013). An evaluation of novel, lower-cost molecular screening tests for human 
Kavita V Anand et al

papillomavirus in rural China. Cancer Prevention Res, 6, 938-8.

\section{(ब) (1) ब}

This work is licensed under a Creative Commons AttributionNon Commercial 4.0 International License. 